Review

\title{
Progress in Understanding the Nature of SS433
}

\section{Anatol Cherepashchuk}

\section{check for}

updates

Citation: Cherepashchuk, A.

Progress in Understanding the

Nature of SS433. Universe 2022, 8, 13.

https://doi.org/10.3390/

universe 8010013

Academic Editors: Nazar R. Ikhsanov,

Galina L. Klimchitskaya and

Vladimir M. Mostepanenko

Received: 26 October 2021

Accepted: 24 December 2021

Published: 27 December 2021

Publisher's Note: MDPI stays neutral with regard to jurisdictional claims in published maps and institutional affiliations.

Copyright: (C) 2021 by the author Licensee MDPI, Basel, Switzerland. This article is an open access article distributed under the terms and conditions of the Creative Commons Attribution (CC BY) license (https:// creativecommons.org/licenses/by/ $4.0 /)$.
P. K. Sternberg State Astronomical Institute, M. V. Lomonosov Moscow State University, Universitetskij Prospect, 13, 119234 Moscow, Russia; cherepashchuk@gmail.com

\begin{abstract}
SS433 is the first example of a microquasar discovered in the Galaxy. It is a natural laboratory for studies of extraordinarily interesting physical processes that are very important for the relativistic astrophysics, cosmic gas dynamics and theory of evolution of stars. The object has been studied for over 40 years in the optical, $X$-ray and radio bands. By now, it is generally accepted that SS433 is a massive eclipsing X-ray binary in an advanced stage of evolution in the supercritical regime of accretion on the relativistic object. Intensive spectral and photometric observations of SS433 at the Caucasian Mountain Observatory of the P. K. Sternberg Astronomical Institute of M. V. Lomonosov Moscow State University made it possible to find the ellipticity of the SS433 orbit and to discover an increase in the system's orbital period. These results shed light on a number of unresolved issues related to SS433. In particular, a refined estimate of the mass ratio $\frac{M_{x}}{M_{v}}>0.8$ was obtained $\left(M_{x}\right.$ and $M_{v}$ are the masses of the relativistic object and optical star). Based on these estimates, the relativistic object in the SS433 system is the black hole; its mass is $>8 M_{\odot}$. The ellipticity of the orbit is consistent with the "slaved" accretion disc model. The results obtained made it possible to understand why SS433 evolves as the semi-detached binary instead of the common envelope system.
\end{abstract}

Keywords: close binaries; black holes; evolution of binary stars

\section{Introduction}

The unique object SS433 shows in its optical spectrum [1], in addition to the standard emission lines of hydrogen and helium, moving emissions, which move along the spectrum with a period of $162.3 \mathrm{~d}$ [2]. The amplitude of these displacements reaches an enormous value of $\sim 1000 \AA$, which amounts up to tens of thousands of $\mathrm{km} \mathrm{s}^{-1}$ on the scale of velocities. Before the discovery of SS433 in the 1970s [2,3], astronomers had never seen anything like this. Therefore, the nature of object SS433 seemed mysterious; some scientists called SS433 "the enigma of the century". In the earliest press releases, there were even mentions that it was possible that we were observing signals from an extraterrestrial intelligence which was shining on us with a powerful re-configurable laser. Fortunately, this hypothesis did not manage to appear in scientific publications since, in 1979, it turned out $[4,5]$ that the moving lines in the spectrum of SS433 are formed in relativistic $(v \simeq 0.26 c)$ collimated jets that are ejected from some central source and precess with a period of $\approx 164 \mathrm{~d}$. The opening angle of the precession cone is $\approx 20^{\circ}$, and the precession axis is inclined with respect to the line of sight by an angle of $\approx 79^{\circ}$. Although the nature of the central source remained unclear, speculation arose that all of these phenomena were enacted in a binary system $[6,7]$. The binary system model was proposed in 1980 by [8], who measured the Doppler shifts of the narrow components of the stationary hydrogen lines and found a period of $\approx 13.1 \mathrm{~d}$, which testified in favor of the close binary system model. The authors from the analysis of their data, assuming that the radial velocity curve measured by them reflects the orbital motion of the components, came to the conclusion that SS433 is a low-mass binary system consisting of a low-mass optical star $\left(1 M_{\odot}\right)$ and a neutron star. As it turned out later, according to our research, this conclusion turned out to be incorrect.

In 1980, we made photometric observations of SS433 and found that SS433 is an eclipsing binary system [9]. The discovery of optical eclipses in the SS433 system made it 
possible to establish that the radial velocity curve constructed by [8] does not reflect the orbital motion of the components but describes the motion of gas in a gas stream flowing from the Lagrange point L1 onto a relativistic object. Analysis of optical eclipses in SS433, as well as studies of the system's extra eclipse brightness depending on the phase of the precessional period [9], made it possible to construct a self-consistent model of SS433 as a massive X-ray eclipsing binary system at an advanced stage of evolution with an optically bright supercritical accretion disc predicted in [10]. Relativistic jets are ejected from the central parts of the supercritical disc perpendicular to its plane. The plane of the disc is inclined to the orbital plane of the SS433 system at an angle of $20^{\circ}$, the disc precesses with a period of $162.3 \mathrm{~d}$ and relativistic jets track the disc precession. The moving emissions are formed in the outer parts of the jets.

The question arose about the reasons for the disc precession. Back in 1973, Katz proposed a precessing disc model to explain the 35-day X-ray cycle in the Her X-1 system [11]. In 1974, Roberts proposed a slaved precession model for an accretion disc in the Her X-1 system [12]. Even earlier, the idea of the slaved disc was expressed by Shakura [13]. The slaved disc model for SS433 was proposed in 1980 by van den Heuvel et al. [14]. In this model, the axis of rotation of the optical star precesses and the disc tracks this precession. The non-perpendicularity of the axis of rotation of the optical star to the orbital plane may be associated with an asymmetric supernova explosion, which inclined the orbital plane of the binary system relative to the axis of rotation of the star [12,15]. To explain the high and stable velocity of matter in the SS433 jets, a model of radiative acceleration was proposed (the so-called line-looking effect $[16,17])$.

Thus, by the early 1980s, the main features of SS433 as a massive close binary X-ray system in the supercritical accretion regime had been clarified. However, many problems remained unresolved. The massive binary system model for SS433 [9] was confirmed by [18], who plotted the radial velocity curve from the stationary HeII 4686 line and measured the mass function of a relativistic object (the lower limit of the mass of an optical star), which turned out to be $\approx 10 M_{\odot}$.

\section{Unsolved Problems}

Over the past 40 years, the object SS433 has been studied in detail in the optical, IR, $\mathrm{X}$-ray and radio ranges (see, for example, reviews [6,7] where all the necessary references are given). Many new and interesting results were obtained on the object SS433, which turned out to be the first example of a microquasar in our Galaxy. However, the main problems associated with this unique object have been unresolved until recently. Let us list some of them.

1. The nature of the precessional variability of SS433. Which model is applicable to SS433: slaved precessing disc model or slaved disc model?

2. The nature of the accreting relativistic object: a neutron star or a black hole?

3. Why has a common envelope not formed in the SS433 system, which undergoes secondary mass exchange in the thermal evolution time scale, and the system evolves as a semi-detached one?

Recently, new observational data have been obtained on the object SS433, which bring us closer to solving these problems.

\section{Discovery of the Ellipticity of SS433's Orbit: Strong Support for a Slaved Accretion Disc Model}

The slaved accretion disc model proposed in [14] seems to be very attractive since a natural physical justification can be formulated for it. Indeed, a small ( 1\%) asymmetry of a supernova explosion, which accompanied the formation of a relativistic object in a binary system, can turn the orbital plane of the binary system relative to the axis of rotation of the optical star by a significant angle [12,15]. A supernova explosion could also significantly increase the eccentricity of the system's orbit. Under the action of tidal interaction from the side of the resulting relativistic object, the axis of rotation of the optical star begins to 
precess, which leads to the formation of an accretion disc that does not lie in the plane of the system's orbit and precesses with the precession period of the optical star. Since a precessing star has a large mass and a huge rotational moment, its precession is very stable and, as a consequence, the observed precession of the accretion disc should also be stable on average. Our long-term (24 years) spectral observations of SS433 $[19,20]$ using published data indicate the stability of the parameters of the kinematic model SS433 over 40 years. This stability can be considered as an argument in favor of the model of a slaved accretion disc in the SS433 system.

However, until recently, there was one fundamental difficulty in applying the model of a slaved accretion disc to the SS433 system. The orbit of SS433 was considered circular since the secondary minimum on the eclipsing light curve, within the errors associated with significant physical variability of the system, was located approximately in the middle between the two primary minima.

The presence of a circular orbit in the SS433 system during asynchronous rotation of the optical star (the star's rotation axis is not perpendicular to the orbital plane) contradicts the theory of tidal synchronization of stars in binary systems. According to this theory [21,22], the synchronization of axial and orbital rotation due to the dissipation of the energy of orbital motion in dynamic tides should occur before the circularization of the orbit. Therefore, if the orbit of SS433 is circular, then the optical star must be synchronized with the orbital revolution and its rotation axis must be perpendicular to the orbital plane. In this case, the accretion disc should lie in the plane of the system's orbit. The slaved disc model is not applicable in this case. As noted above, a significant initial eccentricity of the orbit could appear in a binary system after a supernova explosion, so the assumption of an initial circular orbit seems unnatural. In this regard, the problem of finding direct signs of the ellipticity of the SS433 orbit arises. Indirect evidence of the ellipticity of the orbit was obtained in $[19,20,23]$. In these works, it was found that the velocity of matter in relativistic jets SS433 is modulated by the orbital period $P_{\text {orb }}=13.082 \mathrm{~d}$. In this regard, a hypothesis was put forward about the possible ellipticity of the SS433 orbit: changes in the distance between the components in an elliptical orbit cause a change in the rate of influx of stellar matter into the accretion disc, which can lead to orbital modulation of the velocity of matter in the jets. Other indirect indications of the ellipticity of the SS433 orbit were obtained in $[24,25]$. In this regard, it is of interest to search for direct evidence of the ellipticity of the SS433 orbit.

During 2018-2021, at the Caucasian Mountain Observatory (KGO) of the GAISH MSU, we carried out intensive photometric monitoring of SS433. Several hundred brightness estimates of SS433 were obtained in BVRI filters during 276 observation nights. These data allowed us, in combination with published photometric observations, to construct a high-precision (including 909 individual observations) mean SS433 light curve in the $V$ band in the phases of the precessional period $P_{\text {prec }}=163.3 \mathrm{~d}$, corresponding to the maximum opening of the accretion disc with respect to the observer [26]. Figure 1 shows this curve. It can be seen that the secondary minimum has a noticeable shift relative to the middle between the two primary minima. Analysis of this displacement and the study of the difference in the widths of the primary and secondary minima performed in [26] made it possible to establish that the eccentricity of the SS433 orbit is $e=0.050 \pm 0.007$, and the longitude of the periastron for the epoch of $\approx 1990$ is $\omega=40^{\circ} \pm 20^{\circ}$. The ellipticity of the SS433 orbit discovered by us is in good agreement with the model of a slaved accretion disc. Both observational facts-the long-term stability of the kinematic model parameters and the ellipticity of the SS433 orbit-strongly support the model of a slaved accretion disc in the SS433 system [14], as well as the hypothesis that the reason that the disc does not lie in the plane orbit is an asymmetric supernova explosion in the system [12,15]. 


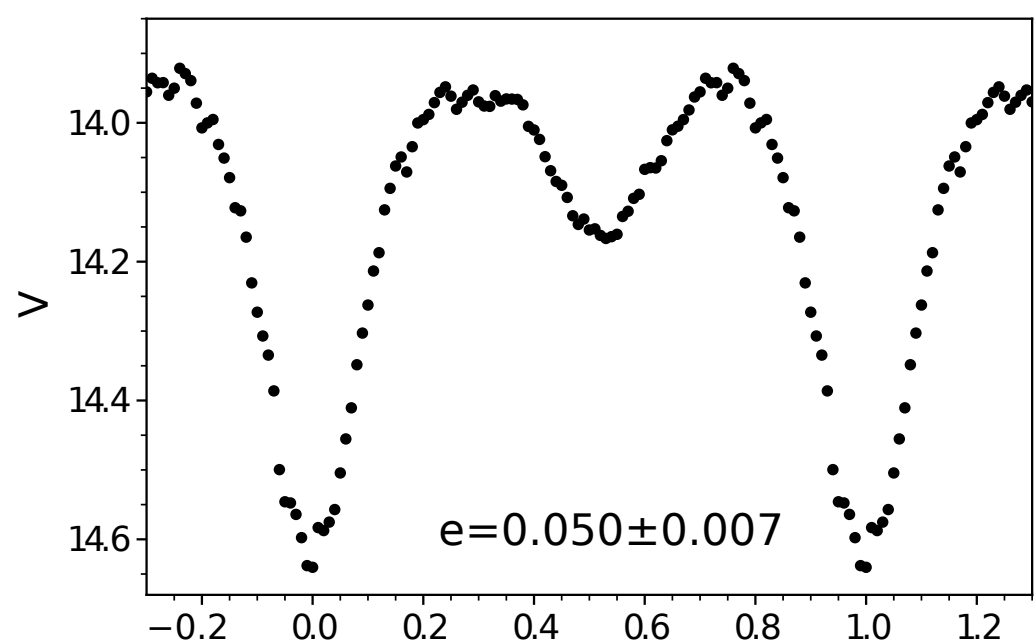

Figure 1. Average orbital light curve of SS433 derived from 909 individual measurements taken during 1978-2012. The observed points were selected in the phases of maximum opening of the accretion disc with respect to the observer. From the paper [26].

\section{The Discovery of an Increase in the Orbital Period of the SS433 System: A Strong Argument for the Presence of a Black Hole}

Despite more than forty years of research, the nature of the relativistic object in the SS433 system remained completely unclear until recently (see discussions on this topic in $[6,7,20])$. In $[19,20]$, using information on the constancy of the orbital period of SS433 over 28 years $[27,28]$, the authors estimated the ratio of the masses of the components in the SS433 $q=\frac{M_{x}}{M_{v}}=0.6\left(M_{x}\right.$ and $M_{v}$ are the masses of a relativistic object and an optical star, respectively). The constancy of the orbital period for the SS433 system is very surprising; after all, the rate of mass loss from the system in the form of wind from the supercritical accretion disc is very high, $\sim 10^{-4} M_{\odot}$ year $^{-1}$ (see, for example, [9]). In [20,29], the balance equations for the loss of angular momentum of the SS433 system were derived by various mechanisms: the transfer of mass and angular momentum during the flow of matter of an optical star through the internal Lagrange point $L_{1}$ to a relativistic object, Jeans mass loss in a symmetric high-velocity outflow matter in the form of wind from the disc as well as the outflow of matter outside the binary system through the outer Lagrange point $L_{2}$. The first mechanism, when flowing from a more massive optical star to a relativistic object, tends to bring the components closer together (and, accordingly, to decrease the orbital period). The second mechanism seeks to increase the distance between the components, that is, to increase the orbital period. The third mechanism leads to a decrease in the distance between the components, i.e., to a decrease in the orbital period. It turns out that, at a huge rate of mass loss in the form of a wind $\sim 10^{-4} M_{\odot}$ year ${ }^{-1}$, an equilibrium between these three mechanisms is established at a very large mass ratio $q \simeq 0.6$. With an optical star mass of $\approx 10 M_{\odot}$ (see, for example, [18]), it follows that the mass of a relativistic object is $6 M_{\odot}$, which is typical of a black hole. In this regard, it is very important to check with additional observations the nature of the constancy or change in the orbital period of SS433. As noted above, in 2018-2021, on the automated 60-cm reflector of the Caucasian Mount Observatory of P. K. Sternberg Astronomical Institute of M. V. Lomonosov Moscow State University, we obtained dense series of photometric observations of SS433 in BVRI filters. With the involvement of all published photometric observational data (since 1979), we discovered an increase in the orbital period of SS433 with time [26]. Figure 2 shows the corresponding plots $O-C$-the differences between the observed moments of the primary minimum of the light curve of SS433 and theoretical moments calculated with linear elements [28]. Here, the parabola corresponds to an increase in the period, and the inclined line corresponds to a constant period (the slope of this line to the abscissa axis determines the correction to a 
constant period). It can be seen that the parabolic approximation of the points in Figure 2 is much more preferable than the linear approximation. Thus, it is shown that the orbital period of SS433 increases. The rate of this increase is $\dot{P}=(1.0 \pm 0.3) \times 10^{-7}$ s per s. It is also shown that the model of the third body in the SS433 system, which can also lead to an increase in the orbital period, is rejected, since the mass of the third body must be more than $16 M_{\odot}$. The lines of such a massive star would be visible in the total spectrum of the SS433 system, which is not observed.

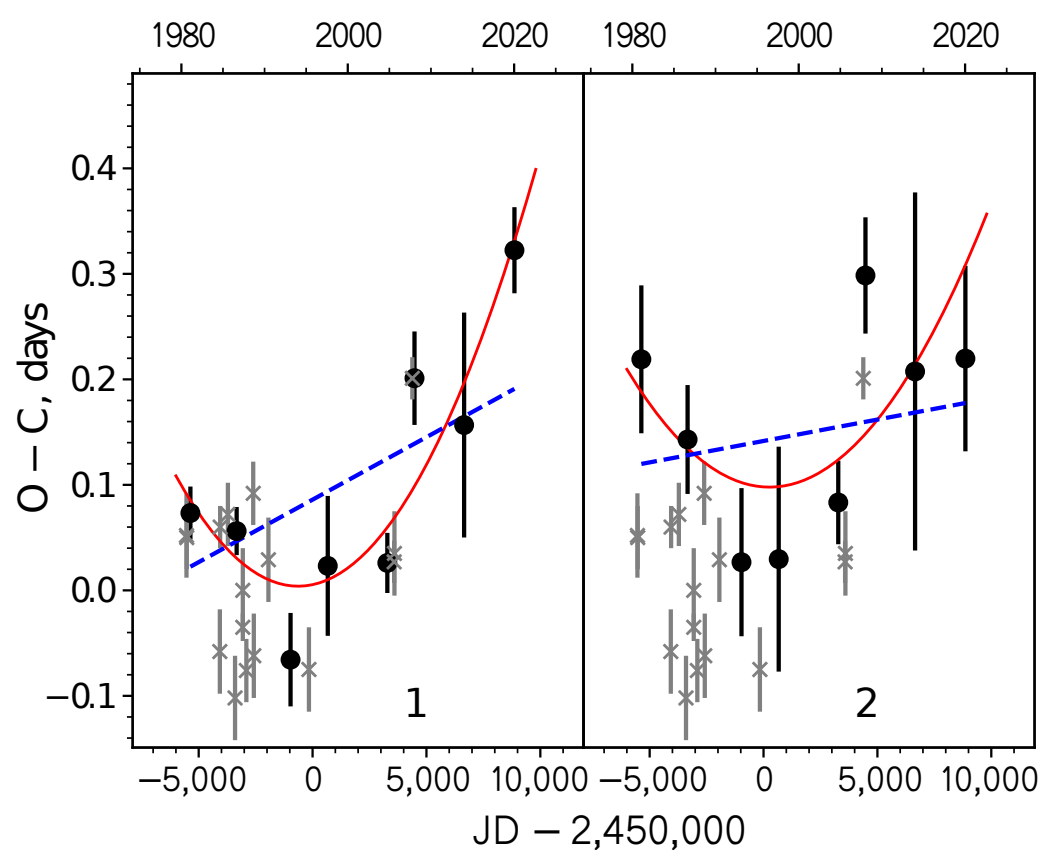

Figure 2. $O-C$ values for SS433, calculated using the ephemeris with a constant orbital period $P_{\text {orb }}=13.08223 \mathrm{~d}$. Right and left panels: $O-C$ calculated by different methods. The parabola corresponds to an increase in the orbital period. The inclined straight line corresponds to a constant period with a $\Delta P$ correction. From the paper [26].

Using the equation to balance the loss of angular momentum and taking into account that the orbital period of SS433 increases, an improved estimate of the mass ratio $q>0.8$ is given in [26]. With the mass of the optical star $M_{v}=10 M_{\odot}$, an estimate is obtained for the mass of the black hole in SS433 $M_{x}>8 M_{\odot}$. Thus, it has been shown that the SS433 system contains a black hole with a mass close to the average mass of stellar black holes in X-ray binaries $\left(\approx 8 \div 10 M_{\odot}\right)$.

The increase in the orbital period of SS433 found by us allows us to reliably reject the presence of a neutron star in the system since, in this case, the orbital period of SS433 should not increase, but should decrease with time, which contradicts observations.

\section{Other Estimates of the Mass Ratio}

The supercritical optically bright accretion disc in the SS433 system makes spectroscopic investigations of the donor star more difficult due to the disc's powerful radiation. Nevertheless, there is a significant progress in this problem by now.

Careful spectroscopic investigations of SS443 made by [30-32] let us estimate the spectral type of the optical star as A7I and to obtain (using absorption lines) the radial velocity curve of this star with the semi-amplitude $K_{\mathrm{v}}=58.2 \pm 3.1 \mathrm{~km} \mathrm{~s}^{-1}$. It corresponds to the optical star's mass function $f_{\mathrm{v}}(m)=0.268 \mathrm{M}_{\odot}$. Similar results were obtained by [33]. In the recent paper by [34], the axial rotation velocity $v_{\text {rot }}=140 \pm 20 \mathrm{~km} \mathrm{~s}^{-1}$ was measured using the Doppler widening of absorption lines of the optical star. Using this value, the corresponding masses and mass ratio of the components of SS433 were estimated: 
$q=\frac{M_{\mathrm{x}}}{M \mathrm{v}}=0.37 \pm 0.04$, the mass of the relativistic object $M_{\mathrm{x}}=4.2 \pm 0.4 M_{\odot}$ and the mass of the optical star $M_{\mathrm{V}}=11.3 \pm 0.6 M_{\odot}$.

There are reasons to suppose that the estimates of the mass of the relativistic object in SS433 based on the spectroscopic data cannot be assumed as reasonably reliable, because the SS433 radiation travel through the intercomponent moving gas medium and also through the rotating circumbinary gas envelope. For the gigantic mass loss rate $\dot{M} \simeq 10^{-4}$ year $^{-1}$, the density of matter in these structures is very high. Therefore, the selective absorption of the optical star's light in moving dense gas structures can significantly distort the orbital radial velocity curve of the donor star measured using absorption lines, see, e.g., [35-37].

The situation with emission lines is also uncertain. For example, the mass function of the relativistic object calculated using the He II 4686 emission line changed from $10 M_{\odot}$ [18] to $2 M_{\odot}$ [38]; it depends on the phase of the precessional period. This can be related with the complicated structure of the wind from the precessing accretion disc whose shape can be asymmetric and twisted.

Because of these problems, great hopes were pinned on studies of X-ray eclipses in the SS433 system. Since the orbital inclination of the system $i=79^{\circ}$ is known from the analysis of displacements of moving emission lines, and the optical star fills or even overfills its inner critical Roche lobe, the analysis of the duration of the X-ray eclipse (when the star hides X-ray structures) gives a principal possibility to estimate the mass ratio of the components.

First such estimate was made by [39-41]. From the analysis of X-ray eclipses in SS433 in the range 2-10 keV in the model of eclipse of the thin relativistic jets (radiating in X-rays) by the optical star, there was obtained a very low mass ratio $q=\frac{M_{\mathrm{x}}}{M_{\mathrm{v}}} \simeq 0.15$. In this case, it was assumed that the optical star fills its Roche lobe.

Nevertheless, there are observational data indicating that the optical star in SS433 not only fills it inner critical Roche lobe but also overfills it. At the same time, the star outflows both through the inner Lagrange point $L_{1}$ and through the outer Lagrange point $L_{2}$. From the analysis of spectral data, arguments in favor of the outflow of the optical star's matter through the outer Lagrange point $L_{2}$ were suggested [42]. It indicates that the star most likely fills its outer Roche lobe whose size is significantly (by 15-20\%) greater than the size of the inner critical Roche lobe. In papers by $[43,44]$, the equatorial outflow of the matter in the plane perpendicular to the direction of the jets was discovered in S433. It also indicates that the star outflows through the $L_{2}$ point and that the star fills its outer Roche lobe. The conclusion about the filling of the outer Roche lobe by the optical star in SS433 and about the outflow through the $L_{2}$ point is supported by the optical and infrared spectroscopy of this object that revealed two-humped stationary hydrogen emission lines $[45,46]$. These lines indicate the presence of the circumbinary envelope which most likely is forming during the outflow of the optical star through the $L_{2}$ point.

Recently, several theoretical studies $[47,48]$ showed that, due to the limited carrying capacity (for the outflowing material) of the surroundings of the $L_{1}$ point, the star (with the radiative envelope) during the mass transfer in the thermal time scale can be in a state of a significant overflow of its inner critical Roche lobe for a long time and the matter can outflow through the $L_{2}$ point.

So, taking into account that the radius of the occulting star in SS433 is significantly greater than the size of the inner critical Roche lobe, the estimate of the component mass ratio obtained by [39-41] should be considered as the lower limit of the mass ratio.

In the review by [7], the results of the interpretation of the eclipsing (orbital) and precessional variability in the hard X-ray range $(k T \approx 18-60 \mathrm{keV})$ were summarized. It was shown that, since the $X$-ray spectrum hardness does not change with the phase of the orbital and precessional variability (the observed $X$-ray flux changes by about five times), the hard $\mathrm{X}$-ray radiation in contradiction to the soft $\mathrm{X}$-ray radiation $(k T \approx 2-10 \mathrm{keV})$ is formed, not in narrow jets but in an extended quasi-isothermal hot corona of the accretion disc. According to [49], the temperature of the corona is $\approx 20 \mathrm{keV}$, its optical depth (Thompson 
scattering) is $\approx 0.2$ and the mass loss rate in the jets is $\dot{M} \approx 3 \times 10^{-7} M_{\odot}$ year $^{-1}$ (the mass loss rate in the wind from the disc is $\sim 10^{-4} M_{\odot}$ year $^{-1}$ ).

In the paper by [7], to analyze the X-ray eclipses and precessional variability of SS433 in the hard X-ray range $(k T=18-60 \mathrm{keV})$, the following model was used: the optical star overflows its inner critical Roche lobe including the case of the filling of the outer Roche lobe, and the star outflows through the $L_{2}$ point. The interpretation of observations was made in the model of precessing accretion disc with hot corona in its central parts. It became clear that $q$ cannot be unambiguously found from the $\mathrm{X}$-ray eclipsing light curve alone. Co-interpretation of both the eclipsing X-ray light curve and precessional light curve in the model of the filling of the outer Roche lobe let us obtain the estimate of the mass ratio $q=\frac{M_{\mathrm{x}}}{M_{\mathrm{V}}}>0.4-0.8$. For the mass of the A7I optical star equal to $10 M_{\odot}$, it gives the estimate of the mass of the black hole $M_{\mathrm{x}} \approx 4-8 M_{\odot}$.

So, the conclusion about the high mass ratio of components in the SS433 system and the presence of the black hole in this system are supported by numerous independent observational data.

\section{Explanation of the Evolutionary Status of SS433 as a Semi-Detached Binary System}

According to the predictions of the modern theory of the evolution of massive close binary systems [50], at the stage of secondary mass exchange, due to the very high rate of the matter flow from an optical star to a relativistic object, a common envelope should form. In this case, due to deceleration by dynamic friction, rapid decreasing of the separation of components occurs. As a result, depending on the initial angular momentum of the binary system, either a short-period WR + c system (of the Cyg X-3 type, $P_{\text {orb }} \simeq 4.8 \mathrm{~h}$ ) is formed or the Thorne-Zhitkov object [51], a completely convective supergiant with relativistic object in the center, is formed. According to [52], the life time for the Thorne-Zhitkov object can be very short due to the neutrino emission.

The SS433 system is at the stage of secondary mass exchange but its evolution, for some reason, took a different path. The system evolves as a semi-detached binary. The removal of mass and angular momentum from the system is not carried out into the common envelope but in the form of a radial outflow of a powerful wind $\left(\dot{M} \simeq 10^{-4} M_{\odot}\right.$ year $^{-1}$, $v \simeq 10^{3} \mathrm{~km} \mathrm{~s}^{-1}$ ) from the supercritical accretion disc. This feature of SS433 has long remained a mystery.

Recently, studies have appeared that have shown that the ratio of the masses of the components is decisive in the evolutionary fate of a massive $\mathrm{X}$-ray binary system at the stage of secondary mass exchange. In a recent work [53], it was shown that when a massive donor star in an X-ray binary system fills or overfills its Roche lobe and outflows onto a relativistic object, then if the component mass ratio is $q=\frac{M_{x}}{M_{\mathcal{v}}} \gtrsim 0.29$, the system can avoid the formation of a common envelope and remain semi-detached. Such a system evolves as a semi-detached system with a stable overflow of the Roche lobe with an optical star and with a stable flow of stellar matter on a relativistic object and with the formation of a supercritical accretion disc with a powerful outflow of stellar wind from it (the so-called isotropic re-emission mode or SS433-like mode). If the mass ratio $q$ is small $(q<0.29)$, then a massive X-ray binary system at the stage of secondary mass exchange inevitably passes through the stage of evolution with a common envelope. The evolutionary scenario that described the formation of the object of SS433 type was considered in the paper by [54].

Thus, the central point in understanding the evolution of SS433 is knowing the real mass ratio of the system's components. Until recently, there was no complete clarity on this issue. Different methods gave estimates from $q \simeq 0.14$ to $q=0.8$ (see reviews by [6,7] for all the necessary references). The new estimate $q>0.8$ [26], obtained on the basis of our discovered increase in the orbital period of SS433, is very reliable and allows us to understand the reason why the SS433 system remains semi-detached. The SS433 system evolves as a semi-detached because the relativistic object here is a relatively large mass black hole $\left(M_{x}>8 M_{\odot}\right)$, which is typical of stellar mass black holes in X-ray binaries. 


\section{Conclusions}

Object SS433 ("Enigma of the century") is the first example of a microquasar discovered in the Galaxy. The object is a natural laboratory where complex and extremely interesting physical processes take place that shed light on many problems: from relativistic astrophysics to the physics and evolution of stars and high energy astrophysics.

For example, recently, the gamma radiation of SS433 was discovered; it can be formed as the result of the interaction of the relativistic jets with the matter of the W50 nebula that surrounds SS433 [55]. In the work by [56], the variability of the gamma flux from SS433, with the period of the precession of the accretion disc, was found. The authors noted that such variability can be related with the formation of the gamma radiation close to the accretion disc of SS433. In this regard, let us note that, in papers by $[57,58]$, in the central part of the supercritical accretion disc in the SS433 system, the hot ( $k T \approx 20 \mathrm{keV})$ extended corona radiating in the hard $(k T=18-60 \mathrm{keV}) \mathrm{X}$-ray range was discovered. Recently, new observational data were obtained that indicated the similarity of physical processes in SS433 and in (still mysterious) objects such as ultra-luminous X-ray sources (ULXs) [59].

In this article, we have summarized the most fundamental problems associated with the object SS433 and showed how the latest observations and theoretical models allow us to understand the nature of this extremely interesting object.

Funding: The work was supported by the Russian Science Foundation grant 17-12-01241 and by the Scientific and Educational School of M. V. Lomonosov Moscow State University "Fundamental and applied space research". The author acknowledges support from the M. V. Lomonosov Moscow State University Program of Development.

Institutional Review Board Statement: Not applicable.

Informed Consent Statement: Not applicable.

Data Availability Statement: The data presented in this study are available on request from the corresponding author.

Conflicts of Interest: The author declares no conflict of interest.

\section{References}

1. Stephenson, C.B.; Sanduleak, N. New H-alpha emission stars in the Milky Way. Astrophys. J. Suppl. Ser. 1977, 33, 459-469. [CrossRef]

2. Margon, B.; Ford, H.C.; Grandi, S.A.; Stone, R.P.S. Enormous periodic Doppler shifts in SS433. Astrophys. J. 1979, 233 , L63-L68. [CrossRef]

3. Clark, D.H.; Murdin, P. An unusual emission-line star/X-ray source radio star, possibly associated with an SNR. Nature 1978, 276, 44-45. [CrossRef]

4. Milgrom, M. On the interpretation of the large variations in the line positions in SS433. AEA 1979, 76, L3-L6.

5. Fabian, A.C.; Rees, M.J. SS 433: A double jet in action? Mon. Not. R. Astron. Soc. 1979, 187, 13P-16P. [CrossRef]

6. Fabrika, S. The jets and supercritical accretion disk in SS433. Astrophys. Space Phys. Res. 2004, 12, 1-152.

7. Cherepashchuk, A.; Postnov, K.; Molkov, S.; Antokhina, E.; Belinski, A. SS433: A massive X-ray binary in an advanced evolutionary stage. New Astron. Rev. 2020, 89, 101542. [CrossRef]

8. Crampton, D.; Cowley, A.P.; Hutchings, J.B. The probable binary nature of SS 433. Astrophys. J. 1980, 235, L131-L135. [CrossRef]

9. Cherepashchuk, A.M. SS 433 as an Eclipsing Binary. Mon. Not. R. Astron. Soc. 1981, 194, 761-769. [CrossRef]

10. Shakura, N.I.; Sunyaev, R.A. Reprint of 1973A\&A....24..337S. Black holes in binary systems. Observational appearance. $A \mathcal{E} A$ 1973, 500, 33-51.

11. Katz, J.I. Thirty-five-day Periodicity in Her X-1. Nat. Phys. Sci. 1973, 246, 87-89. [CrossRef]

12. Roberts, W.J. A slaved disk model for Hercules X-1. Astrophys. J. 1974, 187, 575-584. [CrossRef]

13. Shakura, N.I. Disk Model of Gas Accretion on a Relativistic Star in a Close Binary System. Soviet Ast. $1973,16,756$.

14. van den Heuvel, E.P.J.; Ostriker, J.P.; Petterson, J.A. An early-type binary model for SS 433. AEA 1980, 81, L7-L10.

15. Cherepashchuk, A.M. Longterm Variability of X-ray Binaries-The Aftermath of Supernovae. Sov. Astron. Lett. 1981, 7, 401-403.

16. Shapiro, P.R.; Milgrom, M.; Rees, M.J. Radiative acceleration of astrophysical jets-Line-locking in SS 433. In Extragalactic Radio Sources; Heeschen, D.S., Wade, C.M., Eds.; Cambridge University Press: Cambridge, UK, 1982; Volume 97, p. 209.

17. Shapiro, P.R.; Milgrom, M.; Rees, M.J. The Radiative Acceleration of Astrophysical Jets: Line Locking in SS 433. Astrophys. J. Suppl. Ser. 1986, 60, 393. [CrossRef]

18. Crampton, D.; Hutchings, J.B. The SS 433 binary system. Astrophys. J. 1981, 251, 604-610. [CrossRef] 
19. Davydov, V.V.; Esipov, V.F.; Cherepashchuk, A.M. Spectroscopic monitoring of SS 433: A search for long-term variations of kinematic model parameters. Astron. Rep. 2008, 52, 487-506. [CrossRef]

20. Cherepashchuk, A.M.; Postnov, K.A.; Belinski, A.A. On masses of the components in SS433. Mon. Not. R. Astron. Soc. 2018, 479, 4844-4848. [CrossRef]

21. Zahn, J.P. Reprint of 1977A\&A....57..383Z. Tidal friction in close binary stars. AEA 1977, 500, 121-132.

22. Zahn, J.P. Tidal evolution of close binary stars. I-Revisiting the theory of the equilibrium tide. AEA 1989, 220, 112-116.

23. Blundell, K.M.; Bowler, M.G.; Schmidtobreick, L. Fluctuations and symmetry in the speed and direction of the jets of SS 433 on different timescales. A\&A 2007, 474, 903-910. [CrossRef]

24. Doolin, S.; Blundell, K.M. The Precession of SS433's Radio Ruff on Long Timescales. Astrophys. J. 2009, 698, L23-L26. [CrossRef]

25. Perez, M.S.; Blundell, K.M. Inflow and outflow from the accretion disc of the microquasar SS433: UKIRT spectroscopy. Mon. Not. R. Astron. Soc. 2009, 397, 849-856. [CrossRef]

26. Cherepashchuk, A.M.; Belinski, A.A.; Dodin, A.V.; Postnov, K.A. Discovery of orbital eccentricity and evidence for orbital period increase of SS433. Mon. Not. R. Astron. Soc. 2021, 507, L19-L23. [CrossRef]

27. Cherepashchuk, A.M.; Esipov, V.F.; Dodin, A.V.; Davydov, V.V.; Belinskii, A.A. Spectroscopic Monitoring of SS 433. Stability of Parameters of the Kinematic Model over 40 Years. Astron. Rep. 2018, 62, 747-763. [CrossRef]

28. Goranskij, V. Photometric Mass Estimate for the Compact Component of SS 433: And Yet It Is a Neutron Star. Perem. Zvezdy 2011, 31,5 .

29. Cherepashchuk, A.M.; Postnov, K.A.; Belinski, A.A. Mass ratio in SS433 revisited. Mon. Not. R. Astron. Soc. 2019, 485, $2638-2641$. [CrossRef]

30. Gies, D.R.; Huang, W.; McSwain, M.V. The Spectrum of the Mass Donor Star in SS 433. Astrophys. J. 2002, 578, L67-L70. [CrossRef]

31. Hillwig, T.C.; Gies, D.R.; Huang, W.; McSwain, M.V.; Stark, M.A.; van der Meer, A.; Kaper, L. Identification of the Mass Donor Star's Spectrum in SS 433. Astrophys. J. 2004, 615, 422-431. [CrossRef]

32. Hillwig, T.C.; Gies, D.R. Spectroscopic Observations of the Mass Donor Star in SS 433. Astrophys. J. 2008, 676, L37. [CrossRef]

33. Kubota, K.; Ueda, Y.; Fabrika, S.; Medvedev, A.; Barsukova, E.A.; Sholukhova, O.; Goranskij, V.P. Subaru and Gemini Observations Of SS 433: New Constraint on the Mass of the Compact Object. Astrophys. J. 2010, 709, 1374-1386. [CrossRef]

34. Picchi, P.; Shore, S.N.; Harvey, E.J.; Berdyugin, A. An optical spectroscopic and polarimetric study of the microquasar binary system SS 433. AEA 2020, 640, A96. [CrossRef]

35. Blundell, K.M.; Bowler, M.G.; Schmidtobreick, L. SS 433: Observation of the Circumbinary Disk and Extraction of the System Mass. Astrophys. J. 2008, 678, L47. [CrossRef]

36. Bowler, M.G. Yet more on the circumbinary disk of SS 433. AEA 2013, 556, A149. [CrossRef]

37. Bowler, M.G. SS 433: Two robust determinations fix the mass ratio. AEA 2018, 619, L4. [CrossRef]

38. D'Odorico, S.; Oosterloo, T.; Zwitter, T.; Calvani, M. Evidence that the compact object in SS433 is a neutron star and not a black hole. Nature 1991, 353, 329-331. [CrossRef]

39. Kawai, N.; Matsuoka, M.; Pan, H.C.; Stewart, G.C. GINGA observations of the X-ray eclipse of SS 433. Publ. Astron. Soc. Jpn. $1989,41,491-507$.

40. Brinkmann, W.; Kawai, N.; Matsuoka, M. SS 433-The puzzle continues. A\&A 1989, 218, L13-L16.

41. Kotani, T.; Kawai, N.; Matsuoka, M.; Brinkmann, W. The ASCA Observation Campaign of SS433. In The Hot Universe; Koyama, K., Kitamoto, S., Itoh, M., Eds.; Cambridge University Press: Cambridge, UK, 1998; Volume 188, p. 358.

42. Fabrika, S.N. An extended disc around SS 433. Mon. Not. R. Astron. Soc. 1993, 261, 241-245. [CrossRef]

43. Blundell, K.M.; Mioduszewski, A.J.; Muxlow, T.W.B.; Podsiadlowski, P.; Rupen, M.P. Images of an Equatorial Outflow in SS 433. Astrophys. J. 2001, 562, L79-L82. [CrossRef]

44. Bowler, M.G. More on the circumbinary disk of SS 433. A\&A 2011, 531, A107. [CrossRef]

45. Filippenko, A.V.; Romani, R.W.; Sargent, W.L.W.; Blandford, R.D. Possible Evidence for Disk Emission in SS 433. Astron. J. 1988, 96, 242. [CrossRef]

46. Perez M., S.; Blundell, K.M. SS433's circumbinary ring and accretion disc viewed through its attenuating disc wind. Mon. Not. $R$. Astron. Soc. 2010, 408, 2-8. [CrossRef]

47. Pavlovskii, K.; Ivanova, N. Mass transfer from giant donors. Mon. Not. R. Astron. Soc. 2015, 449, 4415-4427. [CrossRef]

48. Pavlovskii, K.; Ivanova, N.; Belczynski, K.; Van, K.X. Stability of mass transfer from massive giants: Double black hole binary formation and ultraluminous X-ray sources. Mon. Not. R. Astron. Soc. 2017, 465, 2092-2100. [CrossRef]

49. Krivosheyev, Y.M.; Bisnovatyi-Kogan, G.S.; Cherepashchuk, A.M.; Postnov, K.A. Monte Carlo simulations of the broad-band X-ray continuum of SS433. Mon. Not. R. Astron. Soc. 2009, 394, 1674-1684. [CrossRef]

50. Masevich, A.G.; Tutukov, A.V. Ehvolyutsiya Zvezd: Teoriya i Nablyudeniya (Evolution of Stars: Theory and Observations); Nauka: Moscow, Russia, 1988

51. Thorne, K.S.; Zytkow, A.N. Stars with degenerate neutron cores. I. Structure of equilibrium models. Astrophys. J. 1977, 212, 832-858. [CrossRef]

52. Bisnovatyi-Kogan, G.S.; Lamzin, S.A. Stars with Neutron Cores-The Possibility of the Existence of Objects with a Low Neutrino Luminosity. Soviet Ast. 1984, 28, 187-193.

53. van den Heuvel, E.P.J.; Portegies Zwart, S.F.; de Mink, S.E. Forming short-period Wolf-Rayet X-ray binaries and double black holes through stable mass transfer. Mon. Not. R. Astron. Soc. 2017, 471, 4256-4264. [CrossRef] 
54. Bogomazov, A.I. A study of the evolution of the close binaries Cyg X-3, IC 10 X-1, NGC 300 X-1, SS 433, and M33 X-7 using the "scenario machine". Astron. Rep. 2014, 58, 126-138. [CrossRef]

55. Xing, Y.; Wang, Z.; Zhang, X.; Chen, Y.; Jithesh, V. Fermi Observation of the Jets of the Microquasar SS 433. Astrophys. J. 2019, 872, 25. [CrossRef]

56. Rasul, K.; Chadwick, P.M.; Graham, J.A.; Brown, A.M. Gamma-rays from SS433: Evidence for periodicity. Mon. Not. R. Astron. Soc. 2019, 485, 2970-2975. [CrossRef]

57. Cherepashchuk, A.M.; Sunyaev, R.A.; Postnov, K.A.; Antokhina, E.A.; Molkov, S.V. Peculiar nature of hard X-ray eclipse in SS433 from INTEGRAL observations. Mon. Not. R. Astron. Soc. 2009, 397, 479-487. [CrossRef]

58. Cherepashchuk, A.M.; Sunyaev, R.A.; Molkov, S.V.; Antokhina, E.A.; Postnov, K.A.; Bogomazov, A.I. INTEGRAL observations of SS433: System's parameters and nutation of supercritical accretion disc. Mon. Not. R. Astron. Soc. 2013, 436, 2004-2013. [CrossRef]

59. Fabrika, S.; Ueda, Y.; Vinokurov, A.; Sholukhova, O.; Shidatsu, M. Supercritical accretion disks in ultraluminous X-ray sources and SS 433. Nat. Phys. 2015, 11, 551-553. [CrossRef] 